\title{
Local shell-to-shell energy transfer via nonlocal Interactions in fluid turbulence
}

\author{
Mahendra K. Verma, ${ }^{1}$ Arvind Ayyer, ${ }^{2}$ Olivier Debliquy, ${ }^{3}$ Shishir Kumar, ${ }^{4}$ and Amar V. Chandra ${ }^{1}$ \\ ${ }^{1}$ Department of Physics, Indian Institute of Technology, Kanpur 208016, INDIA \\ ${ }^{2}$ Department of Physics and Astronomy, Rutgers University, Piscataway, NJ, USA \\ ${ }^{3}$ Université Libre de Bruxelles, Statistical and Plasma Physics, \\ CP231, Campus Plaine, 1050 Brussels, Belgium \\ ${ }^{4}$ Centre for High Energy Physics, Indian Institute of Science, Bangalore 560012, India
}

(Dated: 1 March 2005)

\begin{abstract}
In this paper we analytically compute the strength of nonlinear interactions in a triad, and the energy exchanges between wavenumber shells in incompressible fluid turbulence. The computation has been done using first-order perturbative field theory. In three dimension, magnitude of triad interactions is large for nonlocal triads, and small for local triads. However, the shell-to-shell energy transfer rate is found to be local and forward. This result is due to the fact that the nonlocal triads occupy much less Fourier space volume than the local ones. The analytical results on three-dimensional shell-to-shell energy transfer match with their numerical counterparts. In two-dimensional turbulence, the energy transfer rates to the near-by shells are forward, but to the distant shells are backward; the cumulative effect is an inverse cascade of energy.
\end{abstract}

Key words: Homogeneous and Isotropic Turbulence, Local transfer.

PACS numbers: 47.27.Ak, 47.27Gs

\section{INTRODUCTION}

Many equations in physics, e.g., Schrodinger equation and diffusion equation, are local in real space. Here, to time-advance a variable at a point, we need the values of the variables and their finite-order derivatives at the same point. It is well known that incompressible Navier-Stokes (NS) equation,

$$
\begin{aligned}
\frac{\partial \mathbf{u}}{\partial t}+(\mathbf{u} \cdot \nabla) \mathbf{u} & =-\nabla p+\nu \nabla^{2} \mathbf{u}, \\
\nabla \cdot \mathbf{u} & =0
\end{aligned}
$$

is nonlocal in real space [1, 2]. Here $\mathbf{u}$ and $p$ are the velocity and pressure fields respectively, and $\nu$ is the kinematic viscosity. The nonlocality is due to the pressure term of Eq. (10), which is obtained by taking the divergence of incompressible Navier-Stokes (NS) equation [2]

$$
\nabla^{2} p=-\nabla \cdot\{\mathbf{u} \cdot \nabla \mathbf{u}\}
$$

Hence,

$$
p(\mathbf{x}, t)=-\int \frac{\nabla^{\prime} \cdot\left\{\mathbf{u}\left(\mathbf{x}^{\prime}, t\right) \cdot \nabla^{\prime} \mathbf{u}\left(\mathbf{x}^{\prime}, t\right)\right\}}{\left|\mathbf{x}-\mathbf{x}^{\prime}\right|},
$$

which is nonlocal because $p(\mathbf{x}, t)$ depends on the velocity field at $\mathbf{x}^{\prime} \neq \mathbf{x}$.

In Fourier space, incompressible NS equation is

$$
\begin{aligned}
\frac{\partial u_{i}(\mathbf{k})}{\partial t}+\nu k^{2} u_{i}(\mathbf{k}) & =-\frac{i}{2} P_{i j m}(\mathbf{k}) \int \frac{d \mathbf{p}}{(2 \pi)^{d}} u_{j}(\mathbf{p}) u_{m}(\mathbf{q}), \\
k_{i} u_{i}(\mathbf{k}) & =0
\end{aligned}
$$

where

$$
P_{i j m}(\mathbf{k})=k_{j}\left(\delta_{i m}-\frac{k_{i} k_{m}}{k^{2}}\right)+k_{m}\left(\delta_{i j}-\frac{k_{i} k_{j}}{k^{2}}\right),
$$

$\mathbf{k}=\mathbf{p}+\mathbf{q}$, and $d$ is the space dimensionality. Note that the factor $-k_{i} k_{j} k_{m} / k^{2}$ of $P_{i j m}(\mathbf{k})$ is due to the pressure term. To determine $\mathbf{u}(\mathbf{k}, t+d t)$ we need the values of field $u_{i}(\mathbf{p})$ where $\mathbf{k}-\mathbf{p}$ could be quite large. Hence, incompressible NS 
equation in nonlocal in Fourier space also [2, 3]. The basic unit of nonlinear interactions in turbulence, called triad interactions, involve three vectors $(\mathbf{k}, \mathbf{p}, \mathbf{q})$ with $\mathbf{k}=\mathbf{p}+\mathbf{q}$. These triad interactions yield energy transfers among the participating modes. The strength of triad interaction is measured using energy exchanges in the triad.

The energy transfers between two wavenumber shells can be computed using the triad interactions. One of the key ingredient of Kolmogorov's phenomenology of turbulence is "local" shell-to-shell energy transfer. That is, maximum energy is transferred from a wavenumber shell to the next wavenumber shell. This observation has been verified in numerical simulations [4, 5]. This result is surprising in view of nonlocal interactions in both real and Fourier space. In this paper, we compute the strength of triad interaction, and shell-to-shell energy transfer in incompressible fluid turbulence using field-theoretic technique.

There have been many attempts in the past to compute the strength of triad interactions and the energy transfers in fluid turbulence. Kraichnan [6] computed these quantities in both 2D and 3D turbulence using "almost Markovian Galilean invariant" turbulence model. He showed the in 3D 35\% of the total energy transfer across a unit wavenumber sphere involves triads in which the smallest wavenumber is more than one-half of the middle wavenumber. Hence, shellto-shell energy transfer in 3D turbulence is local in wavenumber space. Later Domaradzki and Rogallo [5] numerically computed the above quantities and observed that energy transfers in shells are always local, but the triad interactions are nonlocal, i.e., triads having three wavenumbers of very different magnitudes have large magnitudes. This is succinctly described by Domaradzki and Rogallo as "nonlocal interactions and local energy transfer". Domaradzki and Rogallo found their numerical results to be in an excellent agreement with their own Eddy-damped quasi-normal Markovian (EDQNM) calculation. They conjectured that the observed energy transfer as being caused by triads with at least one wavenumber in the energy-containing range. Ohkitani and Kida [7] analyzed the triad interactions carefully and concluded that the nonlocal interaction is strong, but the energy exchange occurs predominantly between comparable scales. They claimed that the third mode of much larger scale is indifferent to the energy transfer as if it were a catalizer in a chemical reaction. Zhou [4] numerically computed the energy transfers using different wavenumber summation scheme, and found the energy transfers to be local. Waleffe [8] did a similar analysis using a decomposition of the velocity field in terms of helical modes. Kishida et al. 9] used wavelet basis to address the same problem and obtained similar results. For review on this topic, refer to Zhou and Speziale [10]. In the present paper we re-look at some of the above conjectures.

In all the above papers, turbulent interactions are measured using a function $S(k \mid p, q)$ (usually called transfer function) that denotes the sum of energy transfers from mode $\mathbf{p}$ and $\mathbf{q}$ to mode $\mathbf{k}$ [11]. Dar et al. [12, 13] point out that the energy transfer from one shell to another shell can not be accurately computed using $S(k \mid p, q)$, essentially because the third mode of the interaction could lie outside both the shells under consideration. To overcome this difficulty, Dar et al. 12] modified the above formulation. They used a new function $S(k|p| q)$, called mode-to-mode energy transfer rate, for the energy transfer from mode $\mathbf{p}$ to mode $\mathbf{k}$, with mode $\mathbf{q}$ acting as a mediator, and showed that the shell-to-shell energy transfer can be correctly computed by this formalism. The computation of $S(k|p| q)$ is done either numerically or using field-theoretic methods. Kishida et al. [9] used similar formalism as Dar et al. [12] for wavelets and numerically computed the shell-to-shell energy transfer rates. In the present paper we quantify the triad interactions using $S(k|p| q)$, and compute them using field-theoretic method. We also calculate the energy transfer rates between wavenumber shells using a first-order perturbation theory. Our analytic arguments justify Domaradzki and Rogallo's 5], Zhou's 4], and Ohkitani and Kida's [7] numerical results that the turbulent interaction is nonlocal, but the shell-to-shell energy transfer is local.

In this paper we also compute mode-to-mode energy transfer rate $S(k|p| q)$ for space dimension other than 2. For $d=2, S(k|p| q)<0$ for most of $p<k$, unlike 3D case. This property of $S(k|p| q)$ is the reason for the inverse energy cascade. We find that the transition from backward to forward energy transfer takes place at $d_{c}=2.25$.

The organization of our paper is as follows: in Sec. 2 we compute $S(k|p| q)$ in the inertial range using first-order field theory. It is shown that nonlinear interactions in incompressible NS are nonlocal. The nature of $S(k|p| q)$ for $2 \mathrm{D}$ and 3D are contrasted. Sec. 3 contains estimates of the shell-to-shell energy transfer for neighbouring and distant shells; since the maximal energy transfer takes place between neighbouring shells, the shell-to-shell energy transfer is said to be local. In Secs. 4 and 5 we compute shell-to-shell energy transfer rates in 3D and 2D respectively. Section 4 also contains a comparison of analytical results with their numerical counterparts. Section 6 contains an elementary discussion on the energy transfer rates in Burgers turbulence. Section 7 contains conclusions.

\section{NONLOCAL INTERACTIONS IN INCOMPRESSIBLE FLUID TURBULENCE}

Kraichnan [6] has computed magnitudes of triad interactions using transfer function $S\left(k^{\prime} \mid p, q\right)\left(\mathbf{k}^{\prime}+\mathbf{p}+\mathbf{q}=0\right)$. In the following discussion we will compute the strength of triad interaction using Dar et al.'s mode-to-mode energy transfer rate $S\left(k^{\prime}|p| q\right)$ [12]

$$
S\left(\mathbf{k}^{\prime}|\mathbf{p}| \mathbf{q}\right)=-\Im\left(\left[\mathbf{k}^{\prime} \cdot \mathbf{u}(\mathbf{q})\right]\left[\mathbf{u}\left(\mathbf{k}^{\prime}\right) \cdot \mathbf{u}(\mathbf{p})\right]\right)
$$




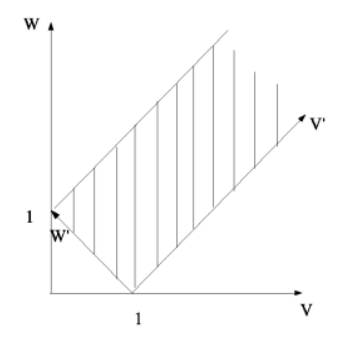

Figure 1: The interacting triad $(\mathbf{k}, \mathbf{p}, \mathbf{q}) / k=(1, v, w)$ under the condition $\mathbf{k}=\mathbf{p}+\mathbf{q}$ is represented by a point $(v, w)$ in the hatched region. The axis $\left(v^{\prime}, w^{\prime}\right)$ are inclined to axis $(v, w)$ by 45 degrees. Note that the local wavenumbers are $v \approx 1, w \approx 1$ or $v^{\prime} \approx w^{\prime} \approx 1 / \sqrt{2}$.

that represents energy transfer mode from $\mathbf{p}$ to mode $\mathbf{k}$, with mode $\mathbf{q}$ acting as a mediator. Here $\Im$ represents the imaginary part of the argument. Here we compute the ensemble average of $S,\left\langle S\left(\mathbf{k}^{\prime}|\mathbf{p}| \mathbf{q}\right)\right\rangle$, using the standard field-theoretic technique [11, 14, 15]. We expand the $\left\langle S\left(\mathbf{k}^{\prime}|\mathbf{p}| \mathbf{q}\right)\right\rangle[$ Eq. 17)] to first order in perturbation (see Verma [13, 16, 17] for details). We assume the flow to be homogeneous and isotropic.

Following the standard field-theoretic procedure, we perform average of $S\left(\mathbf{k}^{\prime}|\mathbf{p}| \mathbf{q}\right)$ and obtain an expression for the energy transfer rate from mode $\mathbf{p}$ to mode $\mathbf{k}$, with mode $\mathbf{q}$ as a mediator

$$
\left.\left\langle S\left(k^{\prime}|p| q\right)\right)\right\rangle=\frac{T_{1}(k, p, q) C(p) C(q)+T_{2}(k, p, q) C(k) C(q)+T_{3}(k, p, q) C(k) C(p)}{\nu(k) k^{2}+\nu(p) p^{2}+\nu(q) q^{2}},
$$

where $C(k)$ is the equal-time correlation function, and $\nu(k)$ is the effective viscosity. The functions $T_{i}(k, p, q)$ are given by

$$
\begin{aligned}
& T_{1}(k, p, q)=k p\left((d-3) z+(d-2) x y+2 z^{3}+2 x y z^{2}+x^{2} z\right), \\
& T_{2}(k, p, q)=-k p\left((d-3) z+(d-2) x y+2 z^{3}+2 x y z^{2}+y^{2} z\right), \\
& T_{3}(k, p, q)=-k q\left(x z-2 x y^{2} z-y z^{2}\right),
\end{aligned}
$$

where $d$ is the space dimensionality, and $x, y, z$ are the cosines of angles between $(\mathbf{p}, \mathbf{q}),(\mathbf{k}, \mathbf{q})$, and (k, p) respectively.

We take Kolmogorov's spectrum for the correlation function, i. e.,

$$
C(k)=\frac{2(2 \pi)^{d}}{S_{d}(d-1)} \frac{K_{K o}|\Pi|^{2 / 3} k^{-5 / 3}}{k^{d-1}},
$$

and renormalized viscosity of McComb and Watt [18] for $\nu(k)$ to be

$$
\nu(k)=\sqrt{K_{K o}} \nu^{*}|\Pi|^{1 / 3} k^{-4 / 3},
$$

where $K_{K o}$ is Kolmogorov's constant, $\Pi$ is the energy flux, and $\nu^{*}$ is a constant related to the renormalized viscosity [18, 19]. Note that in $2 \mathrm{D}$ fluid turbulence, $\Pi$ is negative for the wavenumber region with $5 / 3$ spectral index. McComb and Watt [18, 19] have computed $\nu^{*}$ using renormalization technique. Here we take $K_{K o}=1.6, \nu^{*}=0.38$ for $3 \mathrm{D}$, and $K_{K o}=6.3, \nu^{*}=-0.6$ for $2 \mathrm{D}$.

The interactions are self-similar in the inertial range, which is the region of our interest. Therefore, it is sufficient to analyze $S\left(k^{\prime}|p| q\right)$ for triangles $(1, p / k, q / k)=(1, v, w)$. Since, $|k-p| \leq q \leq k+p,|1-v| \leq w \leq 1+v$, hence any interacting triad $(1, v, w)$ is represented by a point $(v, w)$ in the hatched region of Fig. 1 [1].

The local wavenumbers are $v \approx 1, w \approx 1$, while the rest are called nonlocal wavenumbers. We substitute $C(k)$ and $\nu(k)$ in Eq. (8), which yields

$$
\begin{aligned}
\langle S(v, w)\rangle= & {\left[\frac{4(2 \pi)^{2 d} K_{K o}^{3 / 2} \Pi}{S_{d}^{2}(d-1)^{2} k^{2 d} \nu^{*}}\right] * } \\
& \frac{t_{1}(v, w)(v w)^{-5 / 3-(d-1)}+t_{2}(v, w) w^{-5 / 3-(d-1)}+t_{3}(v, w) v^{-5 / 3-(d-1)}}{1+v^{2 / 3}+w^{2 / 3}}
\end{aligned}
$$



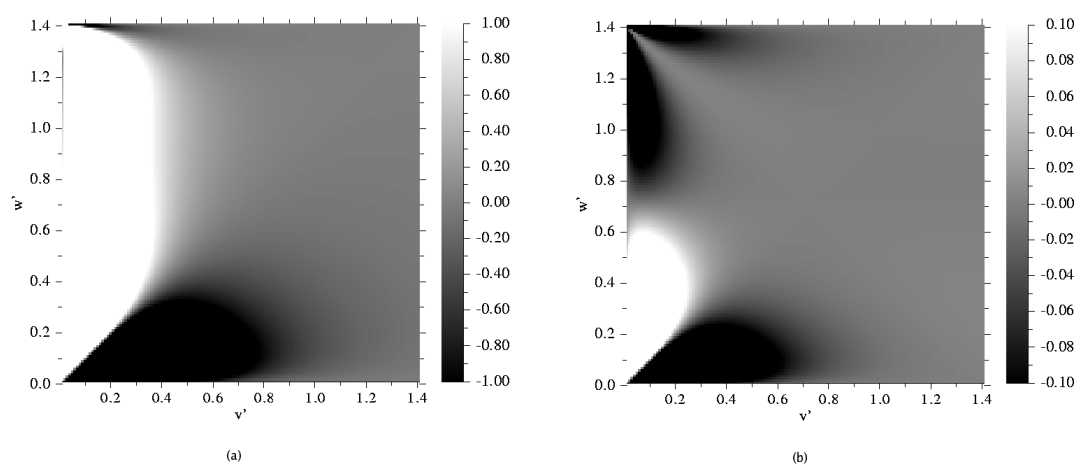

Figure 2: Density plot of $\left\langle S\left(v^{\prime}, w^{\prime}\right)\right\rangle$ of Eq. (8) without the bracketed factor for (a) 3D (b) 2D.

where $t_{i}(v, w)=T_{i}(k, p, q) / k^{2}$. For convenience, $\left\langle S\left(v^{\prime}, w^{\prime}\right)\right\rangle$ are represented in terms of new variables $\left(v^{\prime}, w^{\prime}\right)$ measured from the rotated axis shown in the figure 1 It is easy to show that $v=1+\left(v^{\prime}-w^{\prime}\right) / \sqrt{2}, w=\left(v^{\prime}+w^{\prime}\right) / \sqrt{2}$.

Fig. 2 illustrates density plots of $\left\langle S\left(v^{\prime}, w^{\prime}\right)\right\rangle$ without the bracketed factor. Fig. (a) shows the plot for 3D, while Fig. (b) shows the one for 2D. Note that $\left\langle S\left(v^{\prime}(v, w), w^{\prime}(v, w)\right)\right\rangle$ is the energy transferred from mode $p=v$ to mode $k=1$. In the white region (positive), energy is transferred from mode $p$ to mode $k$, while in the dark regions (negative), mode $p$ receives energy from mode $k$. The value of $S$ at $(v, w)=(1,1)$, or $\left(v^{\prime}, w^{\prime}\right)=(1 / \sqrt{2}, 1 / \sqrt{2})$ is zero in both 2D and 3D.

In $3 \mathrm{D}$ the triads with $v \approx 0, w \rightarrow 1\left(v^{\prime} \approx 0, w^{\prime} \approx \sqrt{2}\right.$ : the top-left corner in $v^{\prime}-w^{\prime}$ plot $)$ have large and positive $\langle S\rangle$, implying that the large wavelength modes give a large amount of energy to the modes near $k \approx 1$. These observations prove that the nonlinear interactions in incompressible NS are nonlocal in Fourier space. In $2 \mathrm{D}$, the triad with $v \approx 0$ have large-negative $\langle S\rangle$ implying that the large wavelength modes take energy from the modes near $k \approx 1$. These observations indicate that the interactions in $2 \mathrm{D}$ turbulence are nonlocal as well, but the large wavelength modes are the sink of energy.

Another common behaviour in both the dimensions is for $w \rightarrow 0, v \approx 1\left(v^{\prime} \approx 0, w^{\prime} \approx 0\right.$, bottom-left corner in $v^{\prime}-w^{\prime}$ plot). Here $\langle S\rangle \gg 0$ for $v<1$, but $\langle S\rangle \ll 0$ for $v>1$. This implies that for these types of triads, the $p$ modes with magnitudes less than $k$ always give energy to the $k$ modes, while the $p$ modes with $p>k$ always take energy from the $k$ modes. When $p, q$ are much larger than $k(v, w \rightarrow \infty),\langle S\rangle$ is small, implying that they interact weakly with $k \approx 1$. In $3 \mathrm{D},\langle S\rangle$ for most of these modes are negative implying that they receive energy from $k=1$. In $2 \mathrm{D}$, however, $\langle S\rangle$ for a large fraction of these triads are positive; hence they supply energy to $k=1$.

The energy cascade is backward in $2 \mathrm{D}$. This is due to the above mentioned backward energy transfer from $k=1$ mode to the smaller wavenumber modes $(\langle S(v \approx 0, w \approx 1)\rangle<0)$, and backward energy transfer from large $v, w$ modes to $k=1$ mode $(\langle S(v, w \gg 1)\rangle>0)$. It is interesting to contrast this behaviour with 3D case where $\langle S(v, w)\rangle$ is somewhat opposite to $2 \mathrm{D}$ case.

The function $\langle S(v, w)\rangle$ in the region with $v \rightarrow 0$ is primarily positive for $d=3$, but is negative for $d=2$. The transition of negative $\langle S\rangle$ to positive $\langle S\rangle$ for the region with $v \rightarrow 0$ occurs near $d_{c}=2.25$. Please refer to Fig. [3(a) for the illustration. It can be shown using field-theoretic calculation that the renormalized viscosity vanishes near $d_{c}=2.25$, and the direction of energy cascade changes from negative to positive at $d=d_{c}$. Fournier and Frisch [20] report $d_{c}=2.05$ which differs a bit from our $d_{c}$. The difference could be because of the fact that Fournier and Frisch [20] use combined energy transfer $S(k \mid p, q)$ for their EDQNM calculation. In Fig. 3(b) we also show the density plot of $\langle S(v, w)\rangle$ for $d=100$. This is a representative illustration for large space dimensions.

The function $\langle S(v, w)\rangle$ can be estimated in the limiting cases using the method given in Appendix of Leslie [1]. In the nonlocal region with $v \rightarrow 0$ (naturally, $w \approx 1$ ),

$$
\langle S(v, w)\rangle \propto v^{\frac{4}{3}-d} .
$$

Clearly, $S(v, w) \rightarrow \infty$ for both 2D and 3D. This observation is consistent with our earlier observation that interactions are nonlocal. However when $v \approx w \approx 1$, we find that

$$
\langle S(v, w)\rangle \propto\left\{\begin{array}{c}
-(v-1) \text { for } d=3 \\
\frac{8}{3}\left[(v-1)^{2}-\frac{1}{2}(w-1)^{2}-\frac{1}{2}(v-1)(w-1)\right] \text { for } d=2
\end{array}\right.
$$



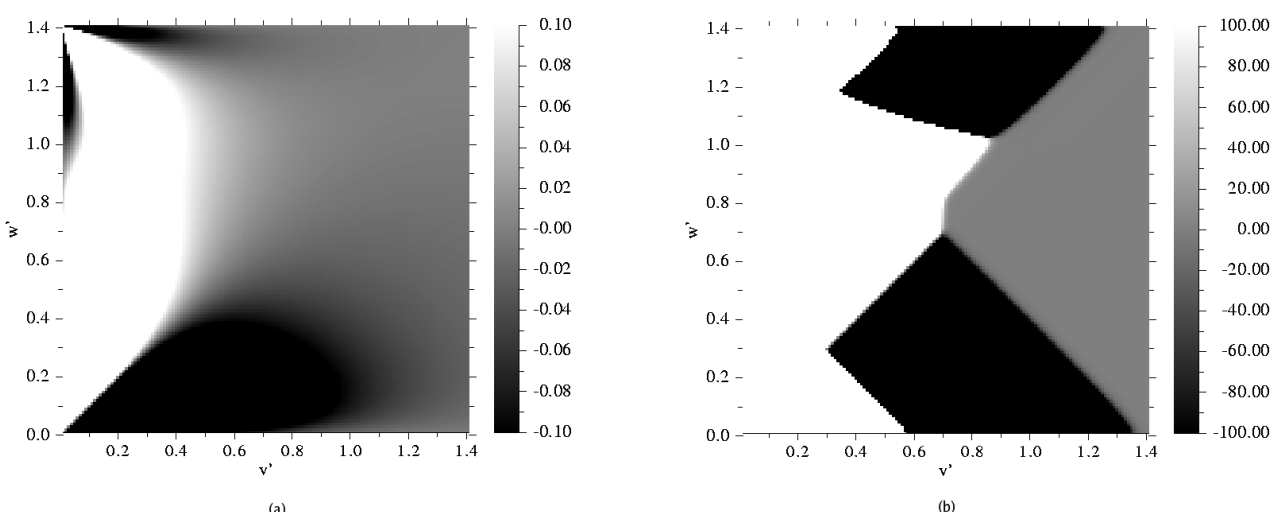

Figure 3: Density plot of $\left\langle S\left(v^{\prime}, w^{\prime}\right)\right\rangle$ of Eq. (8) for (a) $d=2.25$ and (b) $d=100$.

This result shows that the interactions within the local triads $(v \approx 1, w \approx 1)$ are weak. In $3 \mathrm{D}$, among these triads, the ones with $v<1$ or $p<k$ have $\langle S\rangle>0$, hence the energy transfer is from mode $p$ to mode $k$; the sign of $\langle S\rangle$, consequently the sign of energy transfer, is reversed for the modes with $v>1$. However in $2 \mathrm{D}, S$ is somewhat complex in the neighbourhood of $v=w=1$.

For $v \approx 1$ and $w \rightarrow 0$,

$$
\langle S(v, w)\rangle \propto w^{\frac{1}{3}-d},
$$

which again diverges. When $v, w \rightarrow \infty$,

$$
\langle S(v, w)\rangle \propto v^{-\frac{4}{3}-d} .
$$

implying that interactions with large wavenumber modes are weak.

The above estimates are consistent with the graphical plots shown in Figs. 2 and 3 After this discussion, we move on to compute the shell-to-shell energy transfer rates in fluid turbulence.

\section{LOCAL SHELL-TO SHELL ENERGY TRANSFER IN INCOMPRESSIBLE FLUID TURBULENCE}

The wavenumber space is divided into shells $\left(k_{0} s^{n}, k_{0} s^{n+1}\right)$, where $s>1$, and $n$ can take both positive and negative values. The energy transfer rate from $m$ th shell $\left(k_{0} s^{m}, k_{0} s^{m+1}\right)$ to $n$th shell $\left(k_{0} s^{n}, k_{0} s^{n+1}\right)$ is given by [12]

$$
T_{n m}=\sum_{k_{0} s^{n} \leq k \leq k_{0} s^{n+1}} \sum_{k_{0} s^{m} \leq p \leq k_{0} s^{m+1}}\langle S(k|p| q)\rangle .
$$

If the shell-to-Shell energy transfer rate is maximum for the nearest neighbours, and decreases monotonically with the increase of $|n-m|$, then the shell-to-shell energy transfer is said to be local.

If the amplitudes of the Fourier modes $\mathbf{u}(\mathbf{k})$ are available, either from experiments and or from numerical simulations, then we can easily compute the shell-to-shell energy transfer rates using Eqs. (7 18). In this paper, we compute the energy transfer rates between the wavenumbers shells to first order in perturbation [1, 13] that yields

$$
\begin{aligned}
T_{n m}= & \int_{k_{0} s^{n} \leq k \leq k_{0} s^{n+1}} \frac{d \mathbf{k}}{(2 \pi)^{d}} \int_{k_{0} s^{m} \leq p \leq k_{0} s^{m+1}} \frac{d \mathbf{p}}{(2 \pi)^{d}} \\
& \frac{T_{1}(k, p, q) C(p) C(q)+T_{2}(k, p, q) C(k) C(q)+T_{3}(k, p, q) C(k) C(p)}{\nu(k) k^{2}+\nu(p) p^{2}+\nu(q) q^{2}}
\end{aligned}
$$

We nondimensionalize the above equations using [1]

$$
k=\frac{a}{u} ; \quad p=\frac{a}{u} v ; \quad q=\frac{a}{u} w,
$$


where $a$ is an arbitrary constant wavenumber. For our calculation we choose $a=k_{0} s^{n-1}$. Three dimensional integral under the constraint that $\mathbf{k}^{\prime}+\mathbf{p}+\mathbf{q}=0$ is given by [11]

$$
\int_{\mathbf{p}+\mathbf{q}+\mathbf{k}=0} d \mathbf{p}=S_{d-1} \int d p d q\left(\frac{p q}{k}\right)^{d-2}(\sin \alpha)^{d-3}
$$

Using these substitutions, we obtain

$$
\frac{T_{n m}}{|\Pi|}=K_{u}^{3 / 2} \frac{4 S_{d-1}}{(d-1)^{2} S_{d} \nu^{*}} \int_{s^{-1}}^{1} \frac{d u}{u} \int_{u s^{m-n}}^{u s^{m-n+1}} d v \int_{|1-v|}^{1+v} d w(v w)^{d-2}(\sin \alpha)^{d-3} F(v, w),
$$

where $F(v, w)$ is given by

$$
F(v, w)=\frac{t_{1}(v, w)(v w)^{-\frac{2}{3}-d}+t_{2}(v, w) w^{-\frac{2}{3}-d}+t_{3}(v, w) v^{-\frac{2}{3}-d}}{\left(1+v^{2 / 3}+w^{2 / 3}\right)}
$$

with $t_{i}(v, w)=T_{i}(k, k v, k w) / k^{2}$. Eq. (22) provides us with the shell-to-shell energy transfer rates with relative to energy flux $\Pi$. Clearly, $T_{n m}$ depends only on $n-m$, or $T_{n m}=T_{n-i, m-i}$ where $i$ is an integer. Hence, the shell-to-shell energy transfer is self-similar.

Now let us estimate the shell-to-shell energy transfer rates when $m \ll n$. The triads $(1, v, w)$ with $v \rightarrow 0$ participate in this energy transfer. As seen in previous section, for these triads $S(v, w) \propto w^{(4 / 3)-d}$. Therefore,

$$
\begin{aligned}
T_{n m} & \sim \int \frac{d u}{u} \int d v v^{d-2} v^{\frac{4}{3}-d} \int_{|1-v|}^{1+v} d w w^{d-2} \\
& \sim v_{0}^{4 / 3}
\end{aligned}
$$

where $v_{0}$ is the outer radius of shell $m$. Since $v_{0} \rightarrow 0, T_{n m}$ vanishes. Hence, we are able to show that shell-to-shell energy transfer between distant shells in negligible in spite of large interactions between distant wavenumbers. This result is essentially due to small volume of $v$ or $m$-th shell.

We can also compute the shell-to-shell energy transfer rates for close-by shells. Here the participating triads will satisfy $v \approx w \approx 1$. As shown in the earlier section, $S(v, w) \approx-(v-1)$ for these triads. Therefore,

$$
T_{n m} \propto \int \frac{d u}{u} \int d v \int d w(v w)(v-1),
$$

with $n \approx m$. Since our bins are uniform in logarithmic scale, the volume of wavenumber shells is of the order of 1 when $v \approx w \approx 1$. Also the range of $v-1$ is of the order of $v$, which is close to 1 . Therefore $T_{n m}$ will be finite. Hence, the shell-to-shell energy transfer rates between close-by shells is finite. This result is consistent with the local energy transfer assumption of Kolmogorov.

For close-by shells, $p \approx k$, but $q$ can take any value from $|k-p|$ to $k+p$. However, it can be easily shown that the mode $p$ in the triads with $q \ll k$ do not contribute significantly to the shell-to-shell energy transfer. Since, $S(v, w) \propto w^{\frac{1}{3}-d}$ for $w \rightarrow 0$

$$
\begin{aligned}
T_{n m} & \sim \int \frac{d u}{u} \int d \mu \mu \int d w w^{d-2} w^{\frac{1}{3}-d} w \\
& \sim w_{0}^{1 / 3},
\end{aligned}
$$

where $v=1+\mu w$, and $d v=\mu d w$. Clearly the above integral goes to zero. That is, the shell-to-shell energy transfer between close-by shells receive insignificant contribution from the triads satisfying $q \ll p \approx k$.

In this section we showed using the limiting values of $S(k|p| q)$ that the shell-to-shell energy transfer is local in spite of nonlocal interactions among wavenumber modes. However, $T_{n m}$ can be easily computed for the shells in the inertial range. In the next section we will do these calculations.

\section{COMPUTATION OF SHELL-TO-SHELL ENERGY TRANSFER IN 3D}

The shell-to-shell energy transfer rates have been calculated earlier by Ohkitani and Kida [7], and Zhou and Speziale [10] using numerical simulation and EDQNM approximation with $S(k \mid p, q)$. In this section we compute the shell-toshell normalized energy transfer rates $T_{n m} / \Pi$ in three dimensions using Eq. (22). We take $s=2^{1 / 4}$. The integration 


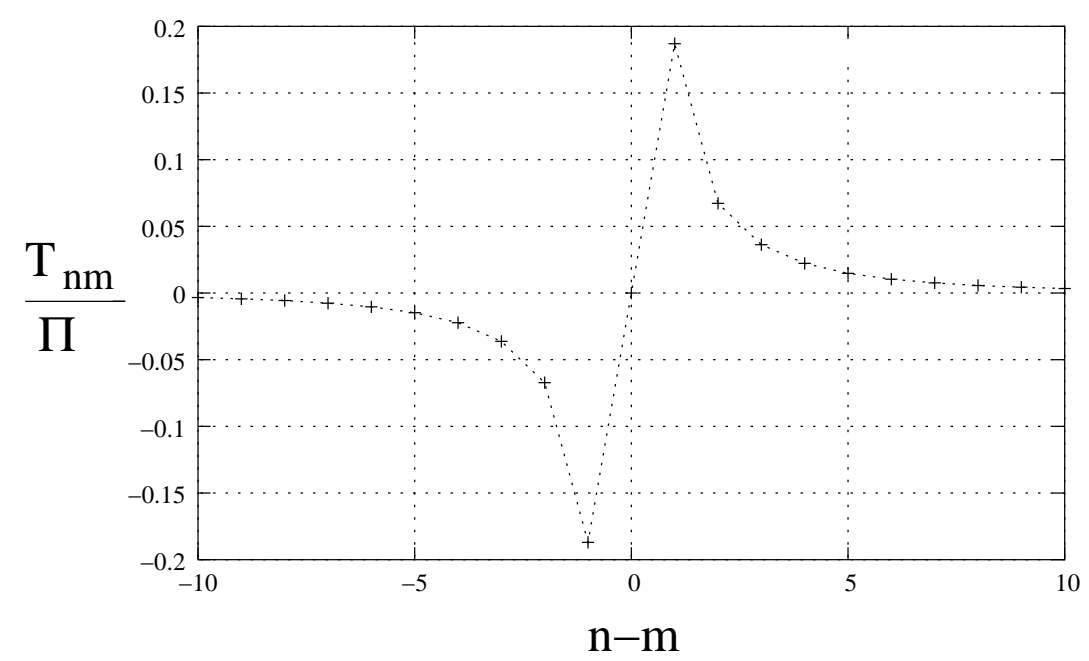

Figure 4: Plot of normalized shell-to-shell energy transfer $T_{n m} / \Pi$ vs $n-m$ for $d=3$. The $n$th shell is $\left(k_{0} s^{n}, k_{0} s^{n+1}\right)$ with $s=2^{1 / 4}$. The energy transfer is maximum for $n=m \pm 1$, hence the energy transfer is local. The energy transfer is also forward.

has been done numerically using Gauss-quadrature method. The constants $\nu^{*}=0.38$ and $K=1.6$ have been taken from McComb and Watt [18] and Verma [13, 19]. The shell-to-shell energy transfer is self-similar, i. e., $T_{n m}$ is function of $n-m$. Therefore we compute $T_{n m} / \Pi$ for various $n-m$. Fig. 4 contains this plot. Note that the shells $m$ and $n$ have been assumed to be inside the inertial range.

From Fig. 4 we can infer that the transfer rates $T_{n m}$ in the inertial range are negative for $n<m$, and positive for $n>m$. Hence a shell gains energy from the smaller wavenumber shells, and loses energy to the higher wavenumber shells. This means that the energy cascades from the smaller wavenumbers to the higher wavenumbers (forward). The most significant energy transfer takes place from $m$ to $m+1$. Hence, the shell-to-shell energy transfer is forward and local, consistent with Kolmogorov's picture of turbulence. Note that the energy transfer is local in spite of nonlocal triad interactions.

To validate our theoretical calculations, we have also computed the shell-to-shell energy transfer rates using the data from Direct Numerical Simulation on a $512^{3}$ grid. The computation was performed when the turbulence was well developed. The Reynold's number using Taylor's microscale was 64.8 , and skewness was -0.54 . We divide the wavenumber space into 15 shells with boundaries at wavenumbers $(2,4,8,11.3,13.5,16,19,22.6,26.9,32,38.1,64,76.1,108,128,256)$. In the inertial range $(k \approx 10-35)$, the shell boundaries are $k_{n}=2^{(n+11) / 4}$. Please refer to Dar et al. [12] for details on numerical procedure. Fig. [5 shows the plots of $T_{n m} / \Pi$ vs. $n-m$ for $m=4 . .9$, which are in the inertial range shells. The plots show self-similarity, local, and forward energy transfer for the inertial range shells. The numerical and theoretical values are in close agreement.

For thicker shells $s=2^{1 / 2}$, the ratio of the smallest to largest wavenumber of the triad is $2 \sqrt{2}$. For these shells, energy transfer to the nearest neighbouring shell is close to $35 \%$. These numbers are consistent with Kraichnan [6] and Zhou's [4] results.

In the next section we will discuss shell-to-shell energy transfer in $2 \mathrm{D}$ turbulence.

\section{SHELL-TO-SHELL ENERGY TRANSFER IN 2D FLUID TURBULENCE}

We compute the shell-to-shell energy transfer in $2 \mathrm{D}$ following the same procedure as given above. The wavenumber range considered is in the inverse cascade regime $\left(E(k) \propto k^{-5 / 3}\right)$. We take $\nu_{*}=-0.6$ and $K=6.3[\underline{6}$, 13]. As shown in Fig. 6. the energy transfer rates from the shell $m$ to the three neighbouring shells $(m+1, m+2, m+3)$ are forward, and the transfers are negative for all shells $n>m+3$. The above result is very similar to Dar et al. [12]'s numerical finding on 2D MHD turbulence (Fig. 10 of Dar et al. [12]). The negative energy transfer from the distant shells are 


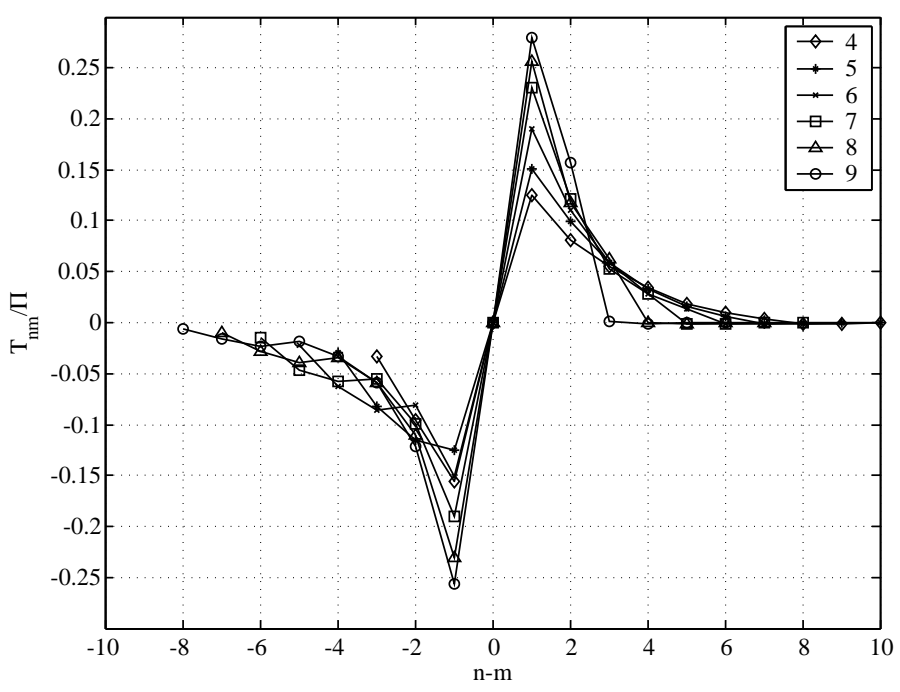

Figure 5: Plots of normalized shell-to-shell energy transfer $T_{n m} / \Pi$ vs. $n-m$ for $m$ from 4 to 9 . The plots collapse on each other indicating self-similarity.

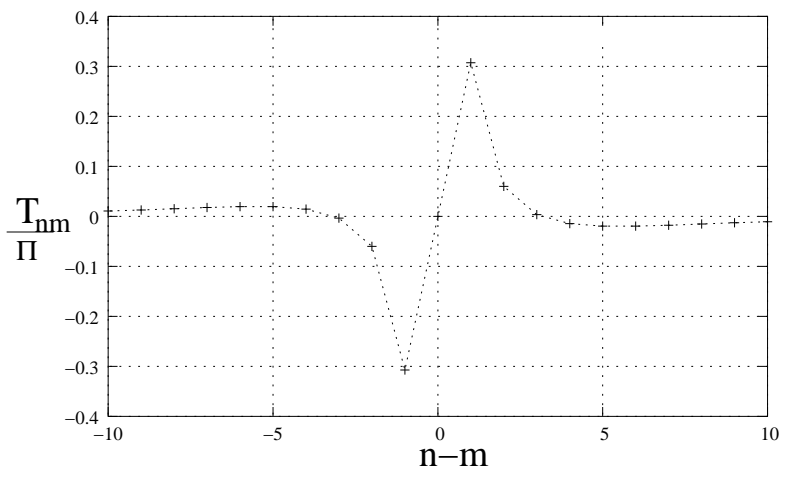

Figure 6: Plot of normalized shell-to-shell energy transfer $T_{n m} /|\Pi|$ vs $n-m$ for $d=2$ in the inertial range. The energy transfer rate from the shell $m$ to the shells $m+1, m+2, m+3$ is forward, but $m+4$ onward it is negative. The net effect of all these transfer is the inverse energy flux $\Pi$.

due to negative $\langle S(v, w)\rangle$ for $v \rightarrow 0$ (top-left of Fig. 2(b)), and positive $\langle S(v, w)\rangle$ for $v, w \gg 1$. The negative $\langle S(v, w)\rangle$ for $v \rightarrow 0$ indicate that large-wavelength modes receive energy from mode $k=1$, and positive $\langle S(v, w)\rangle$ for $v, w \gg 1$ indicate that small-wavelength modes give energy to mode $k=1$. A careful inspection of Fig. 2(b) indicates that the forward energy transfer to shells $(m+1, m+2, m+3)$ is due to a narrow region near $v=w=1$, or $v^{\prime}=w^{\prime}=1 / \sqrt{2}$, where energy transfer is from lower wavenumber to higher number.

The above results on shell-to-shell energy transfer is consistent with the energy flux picture. Note that

$$
\Pi=\sum_{n=m+1}^{\infty}(n-m) T_{n m}
$$

When we perform the $\sum_{n=m+1}^{\infty}(n-m)\left(T_{n m} /|\Pi|\right)$, we obtain -1 , consistent with the inverse cascade of energy in 2D turbulence. 
To summarize, in 2D fluid turbulence, the shell-to-shell energy transfer to the neighbouring shells in forward, but the energy transfer is backward for the distant shells. The above behaviour is due to forward local transfer and backward nonlocal transfers described in Section 2.

In the next section we will contrast the energy transfers in incompressible fluid turbulence with relative to Burgers turbulence (compressible limit).

\section{LOCALITY ISSUES IN BURGERS TURBULENCE}

As discussed in the introduction, Navier-Stokes equation is nonlocal in real space due to pressure. In Burgers equation

$$
\frac{\partial \mathbf{u}}{\partial t}+(\mathbf{u} \cdot \nabla) \mathbf{u}=\nu \nabla^{2} \mathbf{u}
$$

the pressure term is dropped with an implicit assumption that the flow velocity is much greater than sound speed, or the sound speed is very small. This is the opposite limit of incompressible NS where the sound speed is infinite. Hence a very different behaviour is expected for Burgers equation [1]. Clearly, to time-advance the velocity field of Burgers equation at a point, we need local field, and its first and second derivative. Hence, Burgers equation is local in real-space, and nonlocal in Fourier space.

The formula for mode-to-mode energy transfer $S\left(k^{\prime}|p| q\right)$ (Eq. 7 7 ) is not applicable for Burgers equation because it is compressible $(\nabla \cdot \mathbf{u} \neq 0)[12,13]$. Therefore, the shell-to-shell energy transfer cannot be computed accurately. Note however that energy flux can be computed for Burgers equation. The energy flux is multifractal, and $\Pi(k) \propto k^{-1 / 2}$. Therefore, $E(k) \propto \Pi^{2 / 3} k^{-5 / 3}=k^{-2}[1,21]$.

\section{CONCLUSIONS}

It is known that the nonlinear interactions in incompressible Navier-Stokes equation is nonlocal in real space due to the pressure term. In this paper we investigated locality in Fourier space by computing the strength of triad interactions using the formula for the mode-to-mode energy transfer. Our calculation is based on first-order fieldtheoretic technique. We take Kolmogorov's $5 / 3$ powerlaw for the energy spectrum, and the renormalized viscosity for the effective viscosity. It has been shown that the magnitudes of interactions for the nonlocal triads $k \approx p \gg q$ and $k \approx q \gg p$ are large, while the interactions are small for the local triads $k \approx p \approx q$. This result shows that nonlinear interactions in incompressible fluid turbulence is nonlocal in Fourier space as well.

The shell-to-shell energy transfer rates have been investigated by many researchers and ourselves. It is a common wisdom that the shell-to-shell energy transfer is local, that is, maximum energy transfer takes place between nearest shells. We find that local shell-to-shell energy transfer is compatible with the nonlocal triad interactions because the local triads occupy more Fourier space volume as compared to nonlocal $(k \approx q \gg p)$ ones. The local shell-to-shell energy transfer via nonlocal triad interactions is consistent, as seen by Domaradzki and Rogallo [5], Zhou [4], Ohkitani and Kida 7], and Zhou and Speziale [10] in their numerical simulations and EDQNM calculations. We have ourselves computed shell-to-shell energy transfer numerically; our theoretical results match with numerical results very well. In this paper we show this behaviour analytically. The role of the smallest wavenumber mode in the triad is somewhat confusing in earlier papers. We have resolved some of these issues.

We observe interesting behaviour in two dimensions. The shell-to-shell energy transfer rates to the nearby shells are forward, whereas the transfer rates to the far off shells are backward. The net effect is a negative energy flux. This theoretical result is consistent with Dar et al.'s numerical finding [12]. The inverse cascade of energy is consistent with the backward nonlocal energy transfer in mode-to-mode picture $[S(k|p| q)]$. We also show that the transition from backward energy transfer to forward transfer takes place at $d_{c} \approx 2.25$.

The contribution of local triads to the effective or renormalized viscosity has been debated in turbulence literature. In Yakhot and Orszag's [22] renormalization theory, the renormalized viscosity gets contribution from highly nonlocal wavenumber triads. Kraichnan 23, 24] first raised the above objection, and proposed some alternatives. The local energy transfer and and nonlocal triad interaction results discussed in this paper could be of relevance for this issue; this aspect needs further investigation.

To conclude, an application of field-theoretic techniques to turbulence yields interesting results regarding triad interactions and shell-to-shell energy transfers. The method described here has also been applied to magnetohydrodynamic turbulence, and the results are presented in Verma et al. [25]. Further investigations of locality in compressible 
turbulence, and other areas of turbulence will provide us useful clues in furthering our understanding of turbulence.

[1] U. Frisch, Turbulence (Cambridge University Press, Cambridge, 1995).

[2] L. D. Landau and E. M. Lifsitz, Fluid Mechanics (Pergamon Press, Oxford, 1987).

[3] S. A. Orszag, in Fluid Dynamics, Les Houches 1973 Summer School of Theoretical Physics (Gordon and Breach, Berlin, 1977), p. 273

[4] Y. Zhou, Phys. Fluids 5, 1092 (1993).

[5] J. A. Domaradzki and R. S. Rogallo, Phys. Fluids A 2, 413 (1990).

[6] R. H. Kraichnan, J. Fluid Mech. 47, 525 (1971).

[7] K. Ohkitani and S. Kida, Phys. Fluids A 4, 794 (1992).

[8] F. Waleffe, Phys. Fluids A 4, 350 (1992).

[9] K. Kishida, K. Araki, S. Kishiba, and K. Suzuki, Phys. Rev. Lett. 83, 5487 (1999).

[10] Y. Zhou and C. G. Speziale, Appl. Mech. Rev. 51, 267 (1998).

[11] D. C. Leslie, Development in the Theory of Turbulence (Oxford University Press, Claredon, 1973).

[12] G. Dar, M. K. Verma, and V. Eswaran, Physica D 157, 207 (2001).

[13] M. K. Verma, Phys. Rep. 401, 229 (2004).

[14] R. H. Kraichnan, Phys. Fluids 8, 1385 (1965).

[15] W. D. McComb, The Physics of Fluid Turbulence (Oxford University Press, Claredon, 1990).

[16] M. K. Verma, Phys. Rev. E 64, 26305 (2001).

[17] M. K. Verma, Pramana 61, 577 (2003).

[18] W. D. McComb and A. G. Watt, Phys. Rev. A 46, 4797 (1992).

[19] M. K. Verma, Phys. Plasmas 8, 3945 (2001).

[20] J. D. Fournier and U. Frisch, Phys. Rev. A 17, 747 (1979).

[21] M. K. Verma, Physica A 277, 359 (2000).

[22] V. Yakhot and S. A. Orszag, J. Sci. Comput. 1, 3 (1986).

[23] R. H. Kraichnan, Phys. Fluids 30, 1583 (1987).

[24] R. H. Kraichnan, Phys. Fluids 30, 2400 (1987).

[25] M. K. Verma, A. Ayyer, and A. V. Chandra, Phys. Plasmas 12, 82307 (2005). 